\title{
STABLE CONFIGURATIONS OF REPELLING POINTS ON COMPACT MANIFOLDS
}

\author{
BURTON RANDOL
}

(Communicated by Alexander Iosevich)

\begin{abstract}
Using a differential-geometrically intrinsic treatment of counterparts of classical electrostatics introduced by the author in 1984, it is shown that on some compact manifolds, stable configurations of points which mutually repel along all interconnecting geodesics become equidistributed as the number of points increases.
\end{abstract}

In this note, we describe a feature of stable configurations of repelling points on certain manifolds in the sense of 3 (pp. 282-288). Our method is applicable in several contexts, in particular to flat tori and compact hyperbolic manifolds, but we will confine our discussion to the case of compact hyperbolic manifolds since the detailed relevant background material is already available for that case in [3]. The discussion of this subject in [3] was motivated by analogies with classical electrostatics, but the conclusions drawn there can, if preferred, be regarded as being of a formal character, and independent of these analogies. We note that in the flat torus case the analogue of the Selberg pretrace formula is the Poisson summation formula.

Suppose that $M$ is a compact hyperbolic manifold and that $S$ is a finite set of distinct points in $M$. We regard the points of $S$ as mutually repelling along connecting geodesics, with the repelling magnitude varying with distance, as specified by a function $H(\rho), \rho \in[0, \infty)$. In other words, the effect exerted on a point $p \in S$ by points in $S$ is computed by taking the (infinite) vector sum $\sum_{n} H\left(L_{n}\right) V_{n}$, where $L_{n}$ ranges over the lengths of the geodesic segments $g_{n}$ connecting $p$ to points of $S$, and $V_{n}$ is the unit tangent vector to $g_{n}$ at $p$, taken in the repelling direction. Note that $p$ is permitted to act on itself. Conceptually, this can be visualized intrinsically on $M$ or, if one prefers, on the universal covering space $H^{n}$, where the mutual repulsions act along the countably numerous geodesics connecting points, attenuated according to $H(\rho)$. Similar considerations can also be applied to continuous, rather than point distributions on $M$, where the repulsion between infinitesimal parts of $M$ is defined analogously [3] but in this note we will be concerned with finite point distributions. In the interest of expository economy and to avoid unnecessary duplication, we refer the reader to [3] for background material concerning the Selberg pretrace and trace formulas, as well as to the application of the pretrace formula to the matters considered here. In greater detail, following the discussion in [3], we

Received by the editors June 20, 2012 and, in revised form, September 4, 2012.

2010 Mathematics Subject Classification. Primary 37A45.

(C)2014 American Mathematical Society Reverts to public domain 28 years from publication 
assume that $H(\rho)$ is linked to a function $k(\rho)$ by the requirement that $H(\rho)=$ $-k^{\prime}(\rho)$, where $k(\rho)$, which corresponds to a point-pair invariant, is a smooth function on $[0, \infty)$ which vanishes at infinity, and for which the Selberg pretrace formula is valid, with uniform and absolute convergence. As is customary, we denote the Selberg transform of $k(\rho)$ by $h(r)$, and we will be concerned with the values of $h(r)$ on the cross-shaped subset of the complex plane composed of the union $L$ of the real axis with the closed segment of the imaginary axis from $-(n-1) i / 2$ to $(n-1) i / 2$. In the standard parameterization $r \leftrightarrow \lambda$, this corresponds to eigenvalues of the positive Laplacian on $M$, which are situated on $[0, \infty)$. We will also impose, for the purpose of the present note, the additional requirement that $h(r)$ be positive on $L$, and we will call the configuration $S$ stable if the net repelling effect at each point of $S$ is zero.

Let $0=\lambda_{0}<\lambda_{1} \leq \lambda_{2} \cdots$ be the eigenvalues of the positive Laplacian on $M$, with repetitions to account for multiplicity, and suppose $\varphi_{0}, \varphi_{1}, \varphi_{2}, \ldots$ is an associated orthonormal sequence of eigenfunctions. Denote the points of the set $S$ by $x_{1}, \ldots, x_{N}$. With this notation, it follows from the discussion in [3] (cf. especially p. 287), that sets $S$ for which the quantity

$$
\begin{aligned}
& \sum_{n=1}^{\infty}\left(\sum_{i=1}^{N} \sum_{j=1}^{N} \varphi_{n}\left(x_{i}\right) \overline{\varphi_{n}}\left(x_{j}\right)\right) h\left(r_{n}\right) \\
& \quad=\sum_{n=1}^{\infty}\left|\varphi_{n}\left(x_{1}\right)+\ldots+\varphi_{n}\left(x_{N}\right)\right|^{2} h\left(r_{n}\right)
\end{aligned}
$$

is locally minimized in the $N$-fold Cartesian product of $M$ with itself are stable in the above sense. In somewhat more detail, formula (17) from page 283 of [3], which describes the repelling effect produced by a point of $M$ on another point of $M$, can be used to derive this result. The requirement of zero mutual repulsion at each point of $S$ is expressible via that formula as a requirement for the simultaneous vanishing of several instances of (17). This translates, since the gradient on the $N$ fold Cartesian product of $M$ with itself splits into the vector sum of the gradients on the factors, into a necessary condition for (0.1) to have a minimum.

We remark that there is a misprint on page 283 of [3]. Namely, in the formula above (17), the expression $-\nabla_{x} k(x, \gamma y)$ should be replaced by $-\nabla_{x} \sum_{\gamma} k(x, \gamma y)$, and directly above, the words "and by the pretrace formula" should be augmented to "and by the pretrace formula, counting half the $r_{n}$ 's to avoid a factor of $\frac{1}{2}$ ".

These observations suggest, as being of particular interest, the study of the statistical behavior, as $N \rightarrow \infty$, of point configurations that are globally minimizing. As we will now show, such configurations are equidistributed in the limit. In order to see this, we will prove a somewhat more general fact, expressed as a theorem, from which the assertion immediately follows.

Theorem. Suppose that $M$ is a compact Riemannian manifold and $1, \varphi_{1}, \varphi_{2}, \ldots$ is a sequence of continuous functions in $C(M)$, each having $L^{2}$ norm 1 , and with $\int_{M} \varphi_{i}=0$ for $i=1,2, \ldots$. Suppose also that finite linear combinations of functions from the sequence are sup-norm dense in $C(M)$. An important special case in which these conditions are satisfied occurs when the sequence $1, \varphi_{1}, \varphi_{2}, \ldots$ is complete and orthonormal in $L^{2}(M)$. Denote by $M^{N}$ the $N$-fold Cartesian product of $M$ with itself. Let $a_{1}, a_{2}, \ldots$ be a sequence of positive numbers for which the series 
$\sum_{n=1}^{\infty}\left|\varphi_{n}(x)\right|^{2} a_{n}$ is uniformly convergent, and suppose, for $N=1,2,3 \ldots$, that $X_{N} \in M^{N}$ globally minimizes the quadratic expression

$$
\sum_{n=1}^{\infty}\left|\varphi_{n}\left(x_{1}\right)+\ldots+\varphi_{n}\left(x_{N}\right)\right|^{2} a_{n}
$$

Then, as $N \rightarrow \infty$, the sets $X_{N}$ become equidistributed, in the sense that for any continuous function $f$ on $M$,

$$
\frac{1}{N} \sum_{x \in X_{N}} f(x) \rightarrow \int_{M} f
$$

as $N \rightarrow \infty$.

Proof. It follows from the hypotheses that for each $n$,

$$
\int_{M \times \ldots \times M}\left|\varphi_{n}\left(x_{1}\right)+\ldots+\varphi_{n}\left(x_{N}\right)\right|^{2} d x_{1} \ldots d x_{k}=N V^{N-1}
$$

where $V$ is the volume of $M$, so

$$
\int_{M \times \ldots \times M}\left(\sum_{n=1}^{\infty}\left|\varphi_{n}\left(x_{1}\right)+\ldots+\varphi_{n}\left(x_{N}\right)\right|^{2} a_{n}\right) d x_{1} \ldots d x_{k}=N V^{N-1} \sum_{n=1}^{\infty} a_{n} .
$$

In particular, by the mean value theorem for integrals, there exists $\left(\bar{x}_{1}, \ldots, \bar{x}_{N}\right) \in$ $M^{N}$ such that

$$
\sum_{n=1}^{\infty}\left|\varphi_{n}\left(\bar{x}_{1}\right)+\ldots+\varphi_{n}\left(\bar{x}_{N}\right)\right|^{2} a_{n}=\frac{N}{V} \sum_{n=1}^{\infty} a_{n},
$$

which shows that if $\left(p_{1}, \ldots, p_{N}\right) \in M^{N}$ is globally minimizing, then

$$
\sum_{n=1}^{\infty}\left|\varphi_{n}\left(p_{1}\right)+\ldots+\varphi_{n}\left(p_{N}\right)\right|^{2} a_{n} \leq \frac{N}{V} \sum_{n=1}^{\infty} a_{n} .
$$

For a fixed $m$, we therefore conclude from the positivity of the $a_{n}$ 's that

$$
\left|\varphi_{m}\left(p_{1}\right)+\ldots+\varphi_{m}\left(p_{N}\right)\right|^{2} \leq \frac{N}{V} a_{m}^{-1} \sum_{n=1}^{\infty} a_{n},
$$

so

$$
\left|\varphi_{m}\left(p_{1}\right)+\ldots+\varphi_{m}\left(p_{N}\right)\right| \leq \sqrt{\frac{N}{V} a_{m}^{-1} \sum_{n=1}^{\infty} a_{n}},
$$

which implies that

$$
N^{-1}\left|\varphi_{m}\left(p_{1}\right)+\ldots+\varphi_{m}\left(p_{N}\right)\right| \leq \frac{C(m)}{\sqrt{N}}
$$

where

$$
C(m)=\sqrt{\frac{1}{V a_{m}} \sum_{n=1}^{\infty} a_{n}} .
$$

In particular, the $X_{k}$ 's in the limit integrate the uniformly dense set of functions $1, \varphi_{1}, \varphi_{2}, \ldots$ (the constant function is automatic), so Weyl's criterion is satisfied, and the $X_{k}$ 's are equidistributed in the limit. 
Remarks. (1) It is not difficult to give examples of force laws for which $h$ is positive. This is exceptionally easy to see in the hyperbolic 2-dimensional case, since then $k(u)=\frac{1}{2} \hat{h}(u)$, so there are many suitable $h, k$ pairs. In the flat torus case, $k$ and $h$ are in all dimensions Fourier transforms of each other, which again provides many examples. We note also that although it is customary to do so, there is no formal reason to insist that the force law be always repelling.

(2) If we consider globally minimizing configurations to be equivalent when they are mutual images by isometries of $M$, it would be interesting to know something about the behavior with $N$ of the number of inequivalent configurations. In this connection, cf. remark (4).

(3) Our definition of point interaction is intrinsic and does not depend on an isometric embedding of $M$. A prominent example of the latter occurs in the well-known Thomson problem concerning stable configurations of electrons on the unit sphere of $R^{3}$, where the repelling actions resulting from the Coulomb law are transmitted via mutually connecting geodesic segments in the containing space $R^{3}$. Note that the Coulomb law has a singularity at the origin, whereas the methods of this paper require the function $k$ to have a smooth even extension to $R^{1}$ and the series which arise to be suitably convergent. This of course does not rule out the possibility of studying an intrinsic singular law as a limit of regular laws. In view of various isometric embedding theorems, there can be connections between the intrinsic and extrinsic viewpoints. For example, if the distance computed in the containing space depends smoothly on intrinsic distance, the extrinsic case may in some instances become a subcase of the intrinsic case by choosing the intrinsic repelling function $H(\rho)$ to smoothly coincide up to the diameter of $M$ with the repelling function associated with the embedding, then appropriately bringing it down to zero over a distance less than, for example, the injectivity radius of $M$, provided this can be done in such a way that the positivity requirement on $h(r)$ is satisfied. There can, however, be substantial differences between the extrinsic and intrinsic viewpoints. For example, in the case of the unit circle embedded in various ways in $R^{n}$, stable configurations for the embedded problem corresponding to various repelling laws in $R^{n}$ are sensitive to the shape of the embedded circle (cf. 2]), whereas this issue is obviously not present for the intrinsic problem. Some asymptotic and extremal aspects of the intrinsic problem for geodesically equispaced points on a circle are discussed in [4] and [1.

(4) In the intrinsic case, it is possible for a point to act non-trivially on itself. In more detail, it follows from an examination of the analysis leading to formula (17) of [3] that zero-distance action of a point on itself plays no role, but that the effects of the non-trivial geodesics connecting a point to itself are counted. In the flat torus case, these actions cancel out in pairs, and so in that case a single point is stable with respect to itself, but in the hyperbolic case, this is not generally true. In the hyperbolic case, the problem becomes one of studying absolute minima on $M$ of the function

$$
\sum_{n=1}^{\infty}\left|\varphi_{n}(x)\right|^{2} h\left(r_{n}\right), \text { which up to a constant equals } \sum_{\gamma} k(x, \gamma x)
$$


by the pretrace formula. Note that although perhaps generally true, it is not obvious that the number of minima on $M$ of this function is finite. It would be desirable to determine circumstances that ensure this and its multi-point analogues, and thus circumstances which ensure that the question raised in the second remark is meaningful.

\section{REFERENCES}

[1] J. S. Brauchart, D. P. Hardin, and E. B. Saff, Discrete energy asymptotics on a Riemannian circle, Unif. Dist. Theory 7 (2012), no. 2, 77-108. MR.3052946

[2] D. P. Hardin and E. B. Saff, Discretizing manifolds via minimum energy points, Notices Amer. Math. Soc. 51 (2004), no. 10, 1186-1194. MR2104914 (2006a:41049)

[3] B. Randol, The Selberg trace formula (Chapter 11 of Eigenvalues in Riemannian Geometry, by Isaac Chavel), Pure and Applied Mathematics, 115, Academic Press, Orlando, FL, 1984. MR:768584 (86g:58140)

[4] L. Fejes Tóth, Uber eine Punktverteilung auf der Kugel (German, with Russian summary), Acta Math. Acad. Sci. Hungar 10 (1959), 13-19 (unbound insert). MR0105654(21 \#4393)

Ph.D. Program in Mathematics, CUny Graduate Center, 365 Fifth Avenue, New York, New York 10016

E-mail address: brandol@gc.cuny.edu 